ESAIM: COCV 19 (2013) 78-90

DOI: $10.1051 / \mathrm{cocv} / 2011206$
ESAIM: Control, Optimisation and Calculus of Variations

www.esaim-cocv.org

\title{
EQUIVALENT COST FUNCTIONALS AND STOCHASTIC LINEAR QUADRATIC OPTIMAL CONTROL PROBLEMS *
}

\author{
ZHIYONG $\mathrm{YU}^{1}$
}

\begin{abstract}
This paper is concerned with the stochastic linear quadratic optimal control problems (LQ problems, for short) for which the coefficients are allowed to be random and the cost functionals are allowed to have negative weights on the square of control variables. We propose a new method, the equivalent cost functional method, to deal with the LQ problems. Comparing to the classical methods, the new method is simple, flexible and non-abstract. The new method can also be applied to deal with nonlinear optimization problems.
\end{abstract}

Mathematics Subject Classification. 93E20, 49N10, 60H10.

Received October 26, 2010. Revised November 16, 2011.

Published online February 23, 2012.

\section{INTRODUCTION}

In this paper, we are going to investigate a special case of stochastic optimal control problems where the state equations are linear in both the state and control, and the cost functionals are quadratic. Such a control problem is called a linear quadratic stochastic optimal control problem (LQ problem). The LQ problems constitute an extremely important class of optimal control problems, since they can model many problems in applications, and more importantly, many nonlinear control problems can be reasonably approximated by the LQ problems. LQ problems provide a basic knowledge for the general problems. On the other hand, solutions of LQ problems exhibit elegant properties due to their simple and nice structures.

In a deterministic LQ problem, it is well known that the control weight in the cost functional must be positive definite, otherwise the optimization problem would not be well-posed (see Kalman [10], Anderson and Moore [1]). Inspired by this, when people study stochastic LQ problems, the positive definiteness condition for the control weight costs was also assumed, which led to some theories parallel to the deterministic one (see Wonham [17], Davis [7] and Bensoussan [2]). However, it has been pointed out by Peng [12], Chen et al. [6] that the positive definiteness condition seems neither necessary for the infimum of the cost functional being finite, nor for the existence of optimal controls. This reveals one of the significant differences between deterministic

Keywords and phrases. Stochastic LQ problem, stochastic Hamiltonian system, Forward-backward stochastic differential equation, Riccati equation, stochastic maximum principle.

* This work is supported by the National Natural Science Foundation of China (11026185, 11101242), the Natural Science Foundation of Shandong Province, China (ZR2010AQ004), and the Independent Innovation Foundation of Shandong University (2009TS036).

1 School of Economics, Shandong University, Jinan 250100, P.R. China. yuzhiyong@sdu.edu.cn 
and stochastic LQ problems. There are some other literatures focusing on this appealing point, such as Chen and Zhou [5], Chen and Yong [4], Yong and Zhou [19]. In this paper, we will introduce a new method to deal with this issue and obtain some results.

Since the state equation in an LQ problem is linear, by variation of constants formula the state process can be explicitly expressed in terms of the initial state and the control. Substituting this relation into the cost functional, we obtain a functional quadratic in the state and control terms. Thus, the original LQ problem can be transformed to a quadratic optimization problem in a Hilbert space consisting of all square-integrable control processes. However, this functional analysis approach can determine optimal controls only in a very abstract form. The paper [4] used this method. On the other hand, employing the method of completion of squares, one can obtain an optimal control in a linear state feedback form via the so-called stochastic Riccati equation. Along this line, the solvability of the Riccati equations leads to that of the LQ problem. However, it is very difficult to solve the stochastic Riccati equation. We refer to the papers $[5,6]$ for this method.

In the present paper, we introduce a new method called equivalent cost functional method to deal with the stochastic LQ problems with indefinite control weight costs. First of all, we investigate the LQ problems with positive control weight costs, and we call this case standard. As mentioned above, there are many literatures on this case. But in order to completely display the new method, we give a simple treatment for this standard case. Moreover, different from the above classical methods, we study the solvability of the so-called stochastic Hamiltonian system, which is an algebra-differential equation system derived from the stochastic maximum principle. This method was introduced by Peng and $\mathrm{Wu}[14]$ and $\mathrm{Wu}[18]$ to consider a special case of the standard LQ problem studied in this paper. In order to deal with the LQ problem with indefinite control weight cost, we propose a notion of equivalent cost functional. For the same control system, if we use two equivalent cost functionals to criticize control processes respectively, we will get the same results. So the idea is to find an equivalent cost functional with positive control weight cost, and to replace the original cost functional without positive control weight cost. In other words, by means of an equivalent cost functional, we change a non-standard LQ problem to a standard one. Then we can use the results of standard LQ problems to get the results of non-standard ones.

Compared with the classical methods dealing with the LQ problems, the equivalent cost functional method have the following advantages. First, compared with the functional analysis method, the new method is not abstract. The optimal controls are not in abstract forms, but rather are represented by solutions of the stochastic Hamiltonian systems. And the equivalent cost functionals are also defined non-abstract. For example, in Sections 4.1 and 4.2, the equivalent cost functionals are just coming from two simple Itô's formulas respectively. Second, the greatest weakness of the stochastic Riccati equation method is the difficulty to get the solvability of the Riccati equation. The new method can avoid to solve the Riccati equation, by looking for other simpler and more flexible equivalent cost functionals. Third, for the same control system, when we use two equivalent cost functionals respectively, then there exists an invertible linear transformation between the two stochastic Hamiltonian systems. Therefore, by the transformation, we can treat more general stochastic Hamiltonian systems or forward-backward stochastic differential equations (FBSDEs) to get the solvability of them.

The rest of this paper is organized as follows. In Section 2 we introduce some basic notations and formulate the stochastic LQ problems. In Section 3 we use the maximum principle, FBSDE theory and convex analysis to study the standard LQ problems. In Section 4 we introduce the equivalent cost functional method, and use it to deal with the non-standard LQ problems. At the end of this section, we link the new method to the stochastic Riccati equation method and make a comparison between them. In Section 5 we conclude the paper.

\section{Notations AND PROBLEM FORMULATION}

Let $\mathbb{R}^{n}$ be the $n$-dimensional Euclidean space with the usual Euclidean norm $|\cdot|$ and the usual Euclidean inner product $\langle\cdot, \cdot\rangle$. Let $\mathbb{R}^{n \times m}$ be the Hilbert space consisting of all $(n \times m)$ matrices with the inner product

$$
\langle A, B\rangle:=\operatorname{tr}\left\{A B^{\tau}\right\}, \quad \text { for any } A, B \in \mathbb{R}^{n \times m},
$$


where the superscript $\tau$ denotes the transpose of vectors or matrices. Thus, the norm $|A|$ of $A$ induced by the inner product is given by $|A|=\sqrt{\operatorname{tr} A A^{\tau}}$. Particularly, we denote by $\mathcal{S}^{n}$ the set of all $(n \times n)$ symmetric matrices, $\mathcal{S}_{+}^{n}$ the set of all $(n \times n)$ nonnegative definite matrices, and $\hat{\mathcal{S}}_{+}^{n}$ the set of all $(n \times n)$ positive definite matrices.

Next we let $T>0$ be a fixed time horizon and $(\Omega, \mathcal{F}, \mathbb{F}, \mathbb{P})$ be a complete filtered probability space on which is defined a 1-dimensional standard Brownian motion $W(\cdot)$, such that $\mathbb{F}=\left\{\mathcal{F}_{t}\right\}_{t \in[0, T]}$ is the natural filtration of $W(\cdot)$, augmented by all the $\mathbb{P}$-null sets. In other words, we consider only the Brownian filtration throughout this paper.

Remark 2.1. We assume the dimension of Brownian motion $d=1$ just for the simplicity of notations. In fact, all the conclusions in this paper still hold true for the case that the dimension of Browinian motion $d>1$.

For any given Euclidean space or the set of matrices $M$, we denote

- $L_{\mathbb{F}}^{2}(0, T ; M)$ to be the set of all $\mathbb{F}$-adapted $M$-valued processes $X$ such that

$$
\mathbb{E} \int_{0}^{T}|X(t, \omega)|^{2} \mathrm{~d} t<\infty
$$

- $L_{\mathbb{F}}^{\infty}(0, T ; M)$ to be the set of all $\mathbb{F}$-adapted $M$-valued bounded processes;

- $L^{\infty}\left(\Omega, \mathcal{F}_{T}, \mathbb{P} ; M\right)$ to be the set of all $\mathcal{F}_{T}$-measurable $M$-valued bounded random variables.

In the rest of this paper, we shall employ the usual convention of suppressing the $\omega$-dependence of all stochastic processes and random variables.

In this paper, we consider the following linear controlled stochastic differential equation (SDE):

$$
\left\{\begin{array}{l}
\mathrm{d} x(t)=[A(t) x(t)+B(t) u(t)] \mathrm{d} t+[C(t) x(t)+D(t) u(t)] \mathrm{d} W(t) \\
x(0)=x
\end{array}\right.
$$

where $x \in \mathbb{R}^{n}, A(\cdot), C(\cdot) \in L_{\mathbb{F}}^{\infty}\left(0, T ; \mathbb{R}^{n \times n}\right), B(\cdot), D(\cdot) \in L_{\mathbb{F}}^{\infty}\left(0, T ; \mathbb{R}^{n \times m}\right)$. In the above, $u(\cdot)$ is a control process and $x(\cdot)$ is the corresponding state process. We introduce the admissible control set $\mathcal{U}_{a d}=L_{\mathbb{F}}^{2}\left(0, T ; \mathbb{R}^{m}\right)$. Each element of $\mathcal{U}_{a d}$ is called an admissible control. Clearly, for any $u(\cdot) \in \mathcal{U}_{a d}$, there exist a unique solution $x(\cdot) \in L_{\mathbb{F}}^{2}\left(0, T ; \mathbb{R}^{n}\right)$ to equation $(2.1)$, which is called the corresponding admissible state process, and $(x(\cdot), u(\cdot))$ is called an admissible pair. In addition, we are given a quadratic cost functional:

$$
J(u(\cdot))=\frac{1}{2} \mathbb{E} \int_{0}^{T}[\langle R(t) x(t), x(t)\rangle+2\langle S(t) u(t), x(t)\rangle+\langle N(t) u(t), u(t)\rangle] \mathrm{d} t+\frac{1}{2} \mathbb{E}[\langle Q x(T), x(T)\rangle],
$$

where $R(\cdot) \in L_{\mathbb{F}}^{\infty}\left(0, T ; \mathcal{S}^{n}\right), S(\cdot) \in L_{\mathbb{F}}^{\infty}\left(0, T ; \mathbb{R}^{n \times m}\right), N(\cdot) \in L_{\mathbb{F}}^{\infty}\left(0, T ; \mathcal{S}^{m}\right)$ and $Q \in L^{\infty}\left(\Omega, \mathcal{F}_{T}, \mathbb{P} ; \mathcal{S}^{n}\right)$.

Suppose the controller wants to minimize the cost functional $J(u(\cdot))$ by selecting an appropriate admissible control $u(\cdot)$. Then the problem is to find an admissible control $\bar{u}(\cdot) \in \mathcal{U}_{a d}$ such that

$$
J(\bar{u}(\cdot))=\inf _{u(\cdot) \in \mathcal{U}_{a d}} J(u(\cdot)) .
$$

We call the problem above the stochastic linear quadratic optimal control problem. In this paper, we denote it by $L Q$ Problem $(J)$ in order to emphasize the cost functional $J(\cdot)$. If we find an admissible control $\bar{u}(\cdot)$ satisfying (2.3), then we call it an optimal control of LQ Problem $(J)$. The corresponding state process $\bar{x}(\cdot)$ and $(\bar{x}(\cdot), \bar{u}(\cdot))$ are called an optimal state process and an optimal pair, respectively. LQ Problem $(J)$ is called well-posed if $\inf _{u(\cdot) \in \mathcal{U}_{a d}} J(u(\cdot))>-\infty$. 


\section{Results of LQ PROBlem Under STANDARD CONDITIONS}

The stochastic maximum principle is one of the principal approaches to solve stochastic optimization problems. It gives a necessary condition that must be satisfied by any optimal solution. Now we apply the maximum principle to LQ Problem $(J)$.

Lemma 3.1. If $(\bar{x}(\cdot), \bar{u}(\cdot))$ is an optimal pair of LQ Problem $(J)$, then there exists a pair of processes $(\bar{y}(\cdot), \bar{z}(\cdot)) \in L_{\mathbb{F}}^{2}\left(0, T ; \mathbb{R}^{n}\right) \times L_{\mathbb{F}}^{2}\left(0, T ; \mathbb{R}^{n}\right)$ such that $(\bar{x}(\cdot), \bar{u}(\cdot), \bar{y}(\cdot), \bar{z}(\cdot))$ satisfies the following stochastic algebradifferential equation system called a stochastic Hamiltonian system:

$$
\left\{\begin{array}{l}
0=S^{\tau}(t) \bar{x}(t)+B^{\tau}(t) \bar{y}(t)+D^{\tau}(t) \bar{z}(t)+N(t) \bar{u}(t) \\
\mathrm{d} \bar{x}(t)=[A(t) \bar{x}(t)+B(t) \bar{u}(t)] \mathrm{d} t+[C(t) \bar{x}(t)+D(t) \bar{u}(t)] \mathrm{d} W(t) \\
-\mathrm{d} \bar{y}(t)=\left[R(t) \bar{x}(t)+A^{\tau}(t) \bar{y}(t)+C^{\tau}(t) \bar{z}(t)+S(t) \bar{u}(t)\right] \mathrm{d} t-\bar{z}(t) \mathrm{d} W(t) \\
\bar{x}(0)=x, \quad \bar{y}(T)=Q \bar{x}(T)
\end{array}\right.
$$

This lemma is a straightforward consequence of the maximum principle, and we omit the proof. For more details of the maximum principle and the stochastic Hamiltonian system theory, the readers can be referred to the the book by Yong and Zhou [19] and the reference therein.

Lemma 3.1 reveals that LQ Problem $(J)$ is linked to a stochastic Hamiltonian system closely, so the existence and uniqueness of the solution for Hamiltonian system (3.1) are important.

Now we would like to recall an existence and uniqueness result of forward-backward stochastic differential equations (FBSDEs) first obtained by Hu and Peng [8], and then generalized by Peng and Wu [14], Theorem 3.1. This result will be used to get the existence and uniqueness of (3.1). In [14], They studied the following FBSDE:

$$
\left\{\begin{array}{l}
\mathrm{d} x(t)=b(t, x(t), G(t) y(t), H(t) z(t)) \mathrm{d} t+\sigma(t, x(t), G(t) y(t), H(t) z(t)) \mathrm{d} W(t), \\
-\mathrm{d} y(t)=f(t, x(t), y(t), z(t)) \mathrm{d} t-z(t) \mathrm{d} W(t), \\
x(0)=x, \quad y(T)=\Phi(x(T)),
\end{array}\right.
$$

where, $G(\cdot), H(\cdot) \in L_{\mathbb{F}}^{\infty}\left(0, T ; \mathbb{R}^{m \times n}\right)$, for any $(x, y, z) \in \mathbb{R}^{n} \times \mathbb{R}^{n} \times \mathbb{R}^{n}, b(\cdot, x, G(\cdot) y, H(\cdot) z), \sigma(\cdot, x, G(\cdot) y, H(\cdot) z)$, $f(\cdot, x, y, z) \in L_{\mathbb{F}}^{2}\left(0, T ; \mathbb{R}^{n}\right)$ and $\Phi(x) \in L^{2}\left(\Omega, \mathcal{F}_{T}, \mathbb{P} ; \mathbb{R}^{n}\right)$ respectively. They adopted the notations

$$
p=\left(\begin{array}{l}
x \\
y \\
z
\end{array}\right), \quad A(t, p)=\left(\begin{array}{c}
-f(t, x, y, z) \\
b(t, x, G(t) y, H(t) z) \\
\sigma(t, x, G(t) y, H(t) z)
\end{array}\right)
$$

and assumed the following assumptions.

(H1) (Lipschitz condition) $A(t, p)$ and $\Phi(x)$ are uniformly Lipschitz continuous with respect to $p$ and $x$ respectively.

(H2) (Domination condition) There exists a constant $K>0$ such that, for each $x \in \mathbb{R}^{n},\left(y^{i}, z^{i}\right) \in \mathbb{R}^{n} \times \mathbb{R}^{n}$, $i=1,2$,

$$
\left|l\left(t, x, G(t) y^{1}, H(t) z^{1}\right)-l\left(t, x, G(t) y^{2}, H(t) z^{2}\right)\right| \leq K\left|G(t)\left(y^{1}-y^{2}\right)+H(t)\left(z^{1}-z^{2}\right)\right|,
$$

where $l=b$ or $\sigma$.

(H3) (Monotonicity condition) There exists a constant $\nu>0$ such that, for any $p^{i}=\left(x^{i}, y^{i}, z^{i}\right) \in \mathbb{R}^{n} \times \mathbb{R}^{n} \times \mathbb{R}^{n}$, $i=1,2$,

$$
\begin{gathered}
\left\langle A\left(t, p^{1}\right)-A\left(t, p^{2}\right), p^{1}-p^{2}\right\rangle \leq \nu\left|G(t)\left(y^{1}-y^{2}\right)+H(t)\left(z^{1}-z^{2}\right)\right|, \\
\left\langle\Phi\left(x^{1}\right)-\Phi\left(x^{2}\right), x^{1}-x^{2}\right\rangle \geq 0 .
\end{gathered}
$$


Lemma 3.2 (Peng and $\mathrm{Wu}[14])$. Let (H1), (H2) and (H3) hold. Then FBSDE (3.2) admits a unique solution $(x(\cdot), y(\cdot), z(\cdot)) \in L_{\mathbb{F}}^{2}\left(0, T ; \mathbb{R}^{n}\right) \times L_{\mathbb{F}}^{2}\left(0, T ; \mathbb{R}^{n}\right) \times L_{\mathbb{F}}^{2}\left(0, T ; \mathbb{R}^{n}\right)$.

For more results on the theory of FBSDEs, we refer to the book by Ma and Yong [11] and the reference therein. In the rest of this section, we will study LQ Problem $(J)$ under the following standard assumption.

Assumption (STN). $Q \in L^{\infty}\left(\Omega, \mathcal{F}_{T}, \mathbb{P} ; \mathcal{S}_{+}^{n}\right),\left(\begin{array}{cc}R(\cdot) & S(\cdot) \\ S^{\tau}(\cdot) & N(\cdot)\end{array}\right) \in L_{\mathbb{F}}^{\infty}\left(0, T ; \mathcal{S}_{+}^{n+m}\right), N(\cdot) \in L_{\mathbb{F}}^{\infty}\left(0, T ; \hat{\mathcal{S}}_{+}^{m}\right)$. Moreover, the inverse $N^{-1}(\cdot)$ is bounded also.

The basic knowledge on nonnegative and positive matrices leads to the following Remarks 3.3 and 3.4.

Remark 3.3. When $N$ is invertible, $\left(\begin{array}{cc}R(\cdot) & S(\cdot) \\ S^{\tau}(\cdot) & N(\cdot)\end{array}\right) \in L_{\mathbb{F}}^{\infty}\left(\Omega, \mathcal{F}_{T}, \mathbb{P} ; \mathcal{S}_{+}^{n+m}\right)$ is equivalent to $\left(R-S N^{-1} S^{\tau}\right)(\cdot) \in$ $L_{\mathbb{F}}^{\infty}\left(\Omega, \mathcal{F}_{T}, \mathbb{P} ; \mathcal{S}_{+}^{n}\right)$.

Remark 3.4. The following two statements are equivalent:

(i) $N(\cdot)$ is positive and bounded. Moreover, $N^{-1}(\cdot)$ is bounded also;

(ii) there exist two constants $0<k \leq K<\infty$, such that

$$
k|u|^{2} \leq\langle N(t) u, u\rangle \leq K|u|^{2}, \quad \text { for any } u \in \mathbb{R}^{m} \text {, any }(\omega, t) \in \Omega \times[0, T] .
$$

We are now in a position to give the main result of this section.

Theorem 3.5. Under Assumption (STN), the stochastic Hamiltonian system (3.1) admits a unique solution $(\bar{x}(\cdot), \bar{u}(\cdot), \bar{y}(\cdot), \bar{z}(\cdot)) \in L_{\mathbb{F}}^{2}\left(0, T ; \mathbb{R}^{n}\right) \times \mathcal{U}_{\text {ad }} \times L_{\mathbb{F}}^{2}\left(0, T ; \mathbb{R}^{n}\right) \times L_{\mathbb{F}}^{2}\left(0, T ; \mathbb{R}^{n}\right)$. Moreover, $(\bar{x}(\cdot), \bar{u}(\cdot))$ is the unique optimal pair of $L Q$ Problem $(J)$.

Proof. Obviously, Assumption (STN) implies the matrix $N(\cdot)$ is invertible, so we can solve $\bar{u}(\cdot)$ from the algebra equation of the Hamiltonian system (3.1):

$$
\bar{u}(t)=-N^{-1}(t)\left(S^{\tau}(t) \bar{x}(t)+B^{\tau}(t) \bar{y}(t)+D^{\tau}(t) \bar{z}(t)\right) .
$$

Then we substitute (3.3) into the forward and backward differential equations of the Hamiltonian system, and we get an FBSDE:

$$
\left\{\begin{array}{c}
\mathrm{d} \bar{x}(t)=\left[\left(A-B N^{-1} S^{\tau}\right) \bar{x}(t)-B N^{-1} B^{\tau} \bar{y}(t)-B N^{-1} D^{\tau} \bar{z}(t)\right] \mathrm{d} t \\
+\left[\left(C-D N^{-1} S^{\tau}\right) \bar{x}(t)-D N^{-1} B^{\tau} \bar{y}(t)-D N^{-1} D^{\tau} \bar{z}(t)\right] \mathrm{d} W(t), \\
-\mathrm{d} \bar{y}(t)=\left[\left(R-S N^{-1} S^{\tau}\right) \bar{x}(t)+\left(A^{\tau}-S N^{-1} B^{\tau}\right) \bar{y}(t)+\left(C^{\tau}-S N^{-1} D^{\tau}\right) \bar{z}(t)\right] \mathrm{d} t \\
\quad-\bar{z}(t) \mathrm{d} W(t), \\
\bar{x}(0)=x, \quad \bar{y}(T)=Q \bar{x}(T) .
\end{array}\right.
$$

Here and after, we sometimes suppress $t$ also for the matrix-valued processes $A, B$, etc., for the simplicity of notations, whenever no confusion arises. It is obvious (3.1) is equivalent to (3.3) and (3.4). A straightforward calculation shows the coefficients of FBSDE (3.4) satisfy (H1), (H2) and (H3) under Assumption (STN). By Lemma 3.2, FBSDE (3.4) admits a unique solution, and then the Hamiltonian system (3.1) admits a unique solution $(\bar{x}(\cdot), \bar{u}(\cdot), \bar{y}(\cdot), \bar{z}(\cdot)) \in L_{\mathbb{F}}^{2}\left(0, T ; \mathbb{R}^{n}\right) \times \mathcal{U}_{a d} \times L_{\mathbb{F}}^{2}\left(0, T ; \mathbb{R}^{n}\right) \times L_{\mathbb{F}}^{2}\left(0, T ; \mathbb{R}^{n}\right)$.

Next, we are going to show $(\bar{x}(\cdot), \bar{u}(\cdot))$ is an optimal pair of LQ Problem $(J)$. For any given admissible pair $(x(\cdot), u(\cdot))$, we consider the difference between $J(\bar{u}(\cdot))$ and $J(u(\cdot))$ :

$$
\begin{aligned}
J(\bar{u}(\cdot))-J(u(\cdot))= & \frac{1}{2} \mathbb{E} \int_{0}^{T}[\langle R(t) \bar{x}(t), \bar{x}(t)\rangle+2\langle S(t) \bar{u}(t), \bar{x}(t)\rangle+\langle N(t) \bar{u}(t), \bar{u}(t)\rangle] \mathrm{d} t \\
& -\frac{1}{2} \mathbb{E} \int_{0}^{T}[\langle R(t) x(t), x(t)\rangle+2\langle S(t) u(t), x(t)\rangle+\langle N(t) u(t), u(t)\rangle] \mathrm{d} t \\
& +\frac{1}{2} \mathbb{E}[\langle Q \bar{x}(T), \bar{x}(T)\rangle-\langle Q x(T), x(T)\rangle] .
\end{aligned}
$$


Since $Q \in L^{\infty}\left(\Omega, \mathcal{F}_{T}, \mathbb{P} ; \mathcal{S}_{+}^{n}\right)$ and $\left(\begin{array}{cc}R(\cdot) & S(\cdot) \\ S^{\tau}(\cdot) & N(\cdot)\end{array}\right) \in L_{\mathbb{F}}^{\infty}\left(0, T ; \mathcal{S}_{+}^{n+m}\right)$, the functions $\langle Q x, x\rangle$ and $\langle R(t) x, x\rangle+$ $2\langle S(t) u, x\rangle+\langle N(t) u, u\rangle$ are concave on $x$ and $(x, u)$ respectively. By a basic result of convex analysis (see e.g. Rockafellar [15]), we have

$$
\begin{aligned}
J(\bar{u}(\cdot))-J(u(\cdot)) \leq & \frac{1}{2} \mathbb{E} \int_{0}^{T}[\langle R(t) \bar{x}(t), \bar{x}(t)-x(t)\rangle+\langle S(t) \bar{u}(t), \bar{x}(t)-x(t)\rangle \\
& \left.+\left\langle S^{\tau}(t) \bar{x}(t), \bar{u}(t)-u(t)\right\rangle+\langle N(t) \bar{u}(t), \bar{u}(t)-u(t)\rangle\right] \mathrm{d} t \\
& +\frac{1}{2} \mathbb{E}[\langle Q \bar{x}(T), \bar{x}(T)-x(T)\rangle] .
\end{aligned}
$$

Then, applying Itô's formula to $\langle\bar{y}(\cdot), \bar{x}(\cdot)-x(\cdot)\rangle$ on the interval $[0, T]$, we get the right hand side of the above inequality is zero. We get $J(\bar{u}(\cdot))-J(u(\cdot)) \leq 0$. By the arbitrariness of the admissible control $u(\cdot)$, we have $(\bar{x}(\cdot), \bar{u}(\cdot))$ is an optimal pair of LQ Problem $(J)$.

At last, if $(\tilde{x}(\cdot), \tilde{u}(\cdot)) \in L_{\mathbb{F}}^{2}\left(0, T ; \mathbb{R}^{n}\right) \times \mathcal{U}_{a d}$ is also an optimal pair of LQ Problem $(J)$, by Lemma 3.1, there exists $(\tilde{y}(\cdot), \tilde{z}(\cdot)) \in L_{\mathbb{F}}^{2}\left(0, T ; \mathbb{R}^{n}\right) \times L_{\mathbb{F}}^{2}\left(0, T ; \mathbb{R}^{n}\right)$ such that $(\tilde{x}(\cdot), \tilde{u}(\cdot), \tilde{y}(\cdot), \tilde{z}(\cdot))$ satisfies the Hamiltonian system (3.1) also. From the uniqueness of (3.1), we have

$$
(\tilde{x}(\cdot), \tilde{u}(\cdot), \tilde{y}(\cdot), \tilde{z}(\cdot))=(\bar{x}(\cdot), \bar{u}(\cdot), \bar{y}(\cdot), \bar{z}(\cdot))
$$

in the space $L_{\mathbb{F}}^{2}\left(0, T ; \mathbb{R}^{n}\right) \times \mathcal{U}_{a d} \times L_{\mathbb{F}}^{2}\left(0, T ; \mathbb{R}^{n}\right) \times L_{\mathbb{F}}^{2}\left(0, T ; \mathbb{R}^{n}\right)$. We get the uniqueness of optimal pair and complete the proof.

\section{Equivalent COST FUnCtional Method}

When Assumption (STN) doesn't hold true, for example, the control weight cost $N(\cdot)$ is indefinite, it is possible that LQ Problems $(J)$ is well-posed, and the optimal pair exists (see e.g. Peng [12], Chen et al. [6], Chen and Zhou [5], Chen and Yong [4]). In this section, we will introduce a new method, called the equivalent cost functional method, to deal with LQ Problem $(J)$ without Assumption (STN). First, we propose the notion of equivalent cost functional.

Definition 4.1. To a same control system, if there exist two cost functionals $J(\cdot)$ and $\tilde{J}(\cdot)$ satisfying: for any admissible controls $u^{1}(\cdot)$ and $u^{2}(\cdot), J\left(u^{1}(\cdot)\right)<J\left(u^{2}(\cdot)\right)$ if and only if $\tilde{J}\left(u^{1}(\cdot)\right)<\tilde{J}\left(u^{2}(\cdot)\right)$, then we say $\tilde{J}(\cdot)$ is equivalent to $J(\cdot)$.

Remark 4.2. It is easy to see the following two statements are equivalent:

(1) the cost functional $\tilde{J}(\cdot)$ is equivalent to $J(\cdot)$;

(2) for any admissible controls $u^{1}(\cdot)$ and $u^{2}(\cdot)$,

(i) $J\left(u^{1}(\cdot)\right)<J\left(u^{2}(\cdot)\right)$ if and only if $\tilde{J}\left(u^{1}(\cdot)\right)<\tilde{J}\left(u^{2}(\cdot)\right)$;

(ii) $J\left(u^{1}(\cdot)\right)=J\left(u^{2}(\cdot)\right)$ if and only if $\tilde{J}\left(u^{1}(\cdot)\right)=\tilde{J}\left(u^{2}(\cdot)\right)$;

(iii) $J\left(u^{1}(\cdot)\right)>J\left(u^{2}(\cdot)\right)$ if and only if $\tilde{J}\left(u^{1}(\cdot)\right)>\tilde{J}\left(u^{2}(\cdot)\right)$.

Obviously, when we use two equivalent cost functionals $J(\cdot)$ and $\tilde{J}(\cdot)$ to criticize a same control system, we will get the same results. Especially, for LQ Problem $(J)$ and LQ Problem $(\tilde{J})$, the existence and uniqueness of the optimal pairs are equivalent, and the optimal pairs are the same.

The idea to deal with LQ Problem $(J)$ without Assumption (STN) is: if we can find a cost functional $\tilde{J}(\cdot)$ equivalent to the original $J(\cdot)$, and the matrices in $\tilde{J}(\cdot)$ satisfy Assumption (STN), then we can study LQ Problem $(J)$ through studying LQ Problem $(\tilde{J})$. Variously equivalent cost functionals provide a new and flexible approach to deal with LQ Problem $(J)$ without Assumption (STN). 


\subsection{A family of equivalent cost functionals}

As usual, we denote by $C^{1}(0, T ; \mathbb{R})$ the collection of functions $f:[0, T] \rightarrow \mathbb{R}$ which is continuously differential. For any $f \in C^{1}(0, T ; \mathbb{R})$, we apply Itô's formula to $f(\cdot)|x(\cdot)|^{2}$ on the interval $[0, T]$ :

$$
\begin{aligned}
-f(0)|x|^{2}= & -\mathbb{E}\left[f(T)|x(T)|^{2}\right]+\mathbb{E} \int_{0}^{T}\left[\left\langle\left(f^{\prime}(t) I+f(t)\left(A+A^{\tau}+C^{\tau} C\right)\right) x(t), x(t)\right\rangle\right. \\
& \left.+2\left\langle f(t)\left(B+C^{\tau} D\right) u(t), x(t)\right\rangle+\left\langle f(t) D^{\tau} D u(t), u(t)\right\rangle\right] \mathrm{d} t,
\end{aligned}
$$

where $I$ denotes the identity matrix. The constant $-f(0)|x|^{2}$ is represented by any admissible pair $(x(\cdot), u(\cdot))$ in a quadratic form. So we can add $-f(0)|x|^{2} / 2$ on the original cost functional $J(\cdot)$ to get an equivalent one $J^{f}(\cdot)$, and this operation may transform $J(\cdot)$ without Assumption (STN) to $J^{f}(\cdot)$ with Assumption (STN). Especially, when $f(t) \geq 0, t \in[0, T]$, the nonnegative item $\left\langle f(t) D^{\tau} D u(t), u(t)\right\rangle$ in (4.1) may transform a nonpositive control weight cost $N(\cdot)$ in $J(\cdot)$ to a positive one in $J^{f}(\cdot)$. Next we will illustrate this idea in details.

For each $f \in C^{1}(0, T ; \mathbb{R})$, we define

$$
\begin{aligned}
J^{f}(u(\cdot)):= & J(u(\cdot))-\frac{f(0)}{2}|x|^{2} \\
= & \frac{1}{2} \mathbb{E} \int_{0}^{T}\left[\left\langle R^{f}(t) x(t), x(t)\right\rangle+2\left\langle S^{f}(t) u(t), x(t)\right\rangle+\left\langle N^{f}(t) u(t), u(t)\right\rangle\right] \mathrm{d} t \\
& +\frac{1}{2} \mathbb{E}\left[\left\langle Q^{f} x(T), x(T)\right\rangle\right],
\end{aligned}
$$

where

$$
\begin{aligned}
R^{f} & =R+f^{\prime}(t) I+f(t)\left(A+A^{\tau}+C^{\tau} C\right), \\
S^{f} & =S+f(t)\left(B+C^{\tau} D\right), \\
N^{f} & =N+f(t) D^{\tau} D, \\
Q^{f} & =Q-f(T) I .
\end{aligned}
$$

Since $J^{f}(\cdot)$ and $J(\cdot)$ differ in only a constant $-f(0)|x|^{2} / 2$, so they are equivalent. In other words, we get a family of equivalent cost functionals $J^{f}(\cdot), f \in C^{1}(0, T ; \mathbb{R})$. It is obvious that the original cost functional $J(\cdot)$ is in the family with $f \equiv 0$.

By Lemma 3.1, if $\left(\bar{x}^{f}(\cdot), \bar{u}^{f}(\cdot)\right) \in L_{\mathbb{F}}^{2}\left(0, T ; \mathbb{R}^{n}\right) \times \mathcal{U}_{a d}$ is an optimal pair of LQ Problem $\left(J^{f}\right)$, then there exists a pair of processes $\left(\bar{y}^{f}(\cdot), \bar{z}^{f}(\cdot)\right) \in L_{\mathbb{F}}^{2}\left(0, T ; \mathbb{R}^{n}\right) \times L_{\mathbb{F}}^{2}\left(0, T ; \mathbb{R}^{n}\right)$ such that $\left(\bar{x}^{f}(\cdot), \bar{u}^{f}(\cdot), \bar{y}^{f}(\cdot), \bar{z}^{f}(\cdot)\right)$ satisfies the following stochastic Hamiltonian system:

$$
\left\{\begin{array}{l}
0=\left(S^{f}\right)^{\tau} \bar{x}^{f}(t)+B^{\tau} \bar{y}^{f}(t)+D^{\tau} \bar{z}^{f}(t)+N^{f} \bar{u}^{f}(t), \\
\mathrm{d} \bar{x}^{f}(t)=\left[A \bar{x}^{f}(t)+B \bar{u}^{f}(t)\right] \mathrm{d} t+\left[C \bar{x}^{f}(t)+D \bar{u}^{f}(t)\right] \mathrm{d} W(t), \\
-\mathrm{d} \bar{y}^{f}(t)=\left[R^{f} \bar{x}^{f}(t)+A^{\tau} \bar{y}^{f}(t)+C^{\tau} \bar{z}^{f}(t)+S^{f} \bar{u}^{f}(t)\right] \mathrm{d} t-\bar{z}^{f}(t) \mathrm{d} W(t), \\
\bar{x}^{f}(0)=x, \quad \bar{y}^{f}(T)=Q^{f} \bar{x}^{f}(T) .
\end{array}\right.
$$

We notice that (3.1) coincides with (4.3) as $f \equiv 0$. The following lemma shows that there exists a corresponding equivalent relation among the stochastic Hamiltonian systems (4.3).

Lemma 4.3. For all $f \in C^{1}(0, T ; \mathbb{R})$, the existence and uniqueness of Hamiltonian systems (4.3) are equivalent. Moreover, when the Hamiltonian systems (4.3) admit unique solutions $\left(\bar{x}^{f}(\cdot), \bar{u}^{f}(\cdot), \bar{y}^{f}(\cdot), \bar{z}^{f}(\cdot)\right)$, then $\left(\bar{x}^{f}(\cdot), \bar{u}^{f}(\cdot)\right)=(\bar{x}(\cdot), \bar{u}(\cdot))$. 
Proof. We only prove that: for each $f \in C^{1}(0, T ; \mathbb{R})$, the existence and uniqueness of Hamiltonian system (4.3) is equivalent to (3.1).

We notice that if $(\bar{x}(\cdot), \bar{u}(\cdot), \bar{y}(\cdot), \bar{z}(\cdot))$ is a solution of Hamiltonian system (3.1), then

$$
\begin{aligned}
\bar{x}^{f}(\cdot) & =\bar{x}(\cdot) \\
\bar{u}^{f}(\cdot) & =\bar{u}(\cdot) \\
\bar{y}^{f}(\cdot) & =\bar{y}(\cdot)-f(\cdot) \bar{x}(\cdot) \\
\bar{z}^{f}(\cdot) & =\bar{z}(\cdot)-f(\cdot)(C \bar{x}(\cdot)+D \bar{u}(\cdot))
\end{aligned}
$$

solves the Hamiltonian system (4.3). The existence of (3.1) implies that of (4.3). Because the transformation (4.4) is invertible, so the existence of (4.3) implies that of (3.1) also. The equivalence of uniqueness can be derived from the similar argument. From (4.4), when the Hamiltonian systems (4.3) admit unique solutions $\left(\bar{x}^{f}(\cdot), \bar{u}^{f}(\cdot), \bar{y}^{f}(\cdot), \bar{z}^{f}(\cdot)\right)$, then $\left(\bar{x}^{f}(\cdot), \bar{u}^{f}(\cdot)\right)=(\bar{x}(\cdot), \bar{u}(\cdot))$ for any $f \in C^{1}(0, T ; \mathbb{R})$.

Theorem 4.4. If there exists an $f_{0} \in C^{1}(0, T ; \mathbb{R})$ such that, $\left(R^{f_{0}}, S^{f_{0}}, N^{f_{0}}, Q^{f_{0}}\right)$ satisfies Assumption (STN), then for any $f \in C^{1}(0, T ; \mathbb{R})$, the stochastic Hamiltonian system $(4.3)$ admits a unique solution $\left(\bar{x}^{f}(\cdot), \bar{u}^{f}(\cdot)\right.$, $\left.\bar{y}^{f}(\cdot), \bar{z}^{f}(\cdot)\right) \in L_{\mathbb{F}}^{2}\left(0, T ; \mathbb{R}^{n}\right) \times \mathcal{U}_{a d} \times L_{\mathbb{F}}^{2}\left(0, T ; \mathbb{R}^{n}\right) \times L_{\mathbb{F}}^{2}\left(0, T ; \mathbb{R}^{n}\right)$. Moreover, $\left(\bar{x}^{f}(\cdot), \bar{u}^{f}(\cdot)\right)$ is the unique optimal pair of $L Q$ Problem $\left(J^{f}\right)$.

Proof. For $f_{0} \in C^{1}(0, T ; \mathbb{R})$, by Theorem 3.5, the Hamiltonian system (4.3) admits a unique solution $\left(\bar{x}^{f_{0}}(\cdot)\right.$, $\left.\bar{u}^{f_{0}}(\cdot), \bar{y}^{f_{0}}(\cdot), \bar{z}^{f_{0}}(\cdot)\right)$. By Lemma 4.3, for any $f \in C^{1}(0, T ; \mathbb{R})$, the Hamiltonian system (4.3) admits a unique solution $\left(\bar{x}^{f}(\cdot), \bar{u}^{f}(\cdot), \bar{y}^{f}(\cdot), \bar{z}^{f}(\cdot)\right)$, and $\left(\bar{x}^{f}(\cdot), \bar{u}^{f}(\cdot)\right)=\left(\bar{x}^{f_{0}}(\cdot), \bar{u}^{f_{0}}(\cdot)\right)$.

By Theorem 3.5 again, $\left(\bar{x}^{f_{0}}(\cdot), \bar{u}^{f_{0}}(\cdot)\right)$ is the unique optimal pair of LQ Problem $\left(J^{f_{0}}\right)$. Since $J^{f}$ is equivalent to $J^{f_{0}},\left(\bar{x}^{f_{0}}(\cdot), \bar{u}^{f_{0}}(\cdot)\right)=\left(\bar{x}^{f}(\cdot), \bar{u}^{f}(\cdot)\right)$ is also the unique optimal pair of LQ Problem $\left(J^{f}\right)$.

Now, we present two examples to illustrate the result of Theorem 4.4.

Example 4.5. Let the dimension of state process $x(\cdot)$ is 1 . Consider the following LQ problem:

$$
\begin{array}{ll}
\text { Minimize } & J(u(\cdot))=\frac{1}{2} \mathbb{E} \int_{0}^{T}\left[x^{2}(t)+r(t) u^{2}(t)\right] \mathrm{d} t+\frac{1}{2} \mathbb{E}\left[x^{2}(T)\right] \\
\text { Subject to } & \left\{\begin{array}{l}
\mathrm{d} x(t)=u(t) \mathrm{d} W(t), \\
x(0)=x,
\end{array}\right.
\end{array}
$$

where $r(\cdot)$ is an $\mathbb{F}$-adapted bounded real-valued process.

It is easy to see when $r(t)>0, t \in[0, T]$, Assumption (STN) holds true, then LQ Problem $(J)$ admits a unique optimal pair. If we select $f(t)=1+(T-t)$ and use the equivalent cost functional $J^{f}(\cdot)$, then we have $Q^{f}=R^{f}=S^{f}=0$ and $N^{f}=r(t)+1+(T-t)$. If there exists a constant $k>0$, such that $r(t)>-1-(T-t)+k$, $t \in[0, T]$, Assumption (STN) holds true for $\left(Q^{f}, R^{f}, S^{f}, N^{f}\right)$. And then LQ Problem $(J)$ admits a unique optimal pair $(\bar{x}(\cdot), \bar{u}(\cdot)) \in L_{\mathbb{F}}^{2}(0, T ; \mathbb{R}) \times \mathcal{U}_{a d}$, which is defined by the following Hamiltonian system:

$$
\left\{\begin{array}{l}
0=\bar{z}(t)+r(t) \bar{u}(t), \\
\mathrm{d} \bar{x}(t)=\bar{u}(t) \mathrm{d} W(t), \\
-\mathrm{d} \bar{y}(t)=\bar{x}(t) \mathrm{d} t-\bar{z}(t) \mathrm{d} W(t), \\
\bar{x}(0)=x, \quad \bar{y}(T)=\bar{x}(T) .
\end{array}\right.
$$

Moreover we observe that the unique solution of the above Hamiltonian system is $(\bar{x}(t), \bar{u}(t), \bar{y}(t), \bar{z}(t))=$ $(x, 0,-x t+x(T+1), 0), t \in[0, T]$. In other words, the unique optimal pair is $(\bar{x}(\cdot), \bar{u}(\cdot))=(x, 0)$. 
When $r(\cdot)$ is deterministic, the above simple example is introduced by Peng [12], Chen et al. [6]. In [6] and the following paper [5], they studied the LQ problems with indefinite control weight costs by the Riccati equation approach. Since they only obtained the solvability of the Riccati equation when all matrices are deterministic and some other conditions are assumed, then their results can only be used in some special deterministic cases. In contrast, the equivalent cost functional method is more effective in the general random case. In the next example, we will apply the equivalent cost functional method to an LQ problem both with $C \neq 0$ and with random matrices.

Example 4.6. Let $D=I, S=0$ and $N=-\lambda I$, where $\lambda \geq 0$ is a constant. We consider the following LQ problem:

$$
\begin{array}{ll}
\text { Minimize } & J(u(\cdot))=\frac{1}{2} \mathbb{E} \int_{0}^{T}\left[\langle R x(t), x(t)\rangle-\lambda|u(t)|^{2}\right] \mathrm{d} t+\frac{1}{2} \mathbb{E}[\langle Q x(T), x(T)\rangle] \\
\text { Subject to } & \left\{\begin{array}{l}
\mathrm{d} x(t)=[A x(t)+B u(t)] \mathrm{d} t+[C x(t)+u(t)] \mathrm{d} W(t), \\
x(0)=x .
\end{array}\right.
\end{array}
$$

The control weight in the cost functional $N=-\lambda I$ is nonpositive, so Assumption (STN) doesn't hold true for $J(\cdot)$. Now we use the equivalent cost functionals $J^{f}(\cdot)$ to study this LQ problem. If there exist two constants $\lambda<k<K<\infty$ such that $Q \geq K I$, and there exists an $f \in C^{1}(0, T ; \mathbb{R})$ such that $f(T) \in[k, K], f(t) \geq k$ for all $t \in[0, T]$, and

$$
R+f^{\prime}(t) I+f(t)\left(A+A^{\tau}+C^{\tau} C\right)-\frac{f(t)^{2}}{f(t)-\lambda}\left(B+C^{\tau}\right)\left(B^{\tau}+C\right) \geq 0,
$$

then Assumption (STN) holds true for $\left(Q^{f}, R^{f}, S^{f}, N^{f}\right)$. And then LQ Problem $(J)$ admits a unique optimal pair $(\bar{x}(\cdot), \bar{u}(\cdot)) \in L_{\mathbb{F}}^{2}\left(0, T ; \mathbb{R}^{n}\right) \times \mathcal{U}_{a d}$, which is defined by the following Hamiltonian system:

$$
\left\{\begin{array}{l}
0=B^{\tau} \bar{y}(t)+\bar{z}(t)-\lambda \bar{u}(t) \\
\mathrm{d} \bar{x}(t)=[A \bar{x}(t)+B \bar{u}(t)] \mathrm{d} t+[C \bar{x}(t)+\bar{u}(t)] \mathrm{d} W(t) \\
-\mathrm{d} \bar{y}(t)=\left[R \bar{x}(t)+A^{\tau} \bar{y}(t)+C^{\tau} \bar{z}(t)\right] \mathrm{d} t-\bar{z}(t) \mathrm{d} W(t) \\
\bar{x}(0)=x, \quad \bar{y}(T)=Q \bar{x}(T)
\end{array}\right.
$$

It is obvious that this example provides a sufficient condition for this LQ problem with nonpositive control weight cost. But also, by the invertible linear transformation (4.4), we can treat more general FBSDEs to get the existence and uniqueness results of them. Let us explain in details. (i). In the case that $\lambda<0$, the Hamiltonian system can be represented by the following FBSDE (where $\bar{x}(\cdot), \bar{y}(\cdot), \bar{z}(\cdot)$ are the three unknown processes):

$$
\left\{\begin{array}{l}
\mathrm{d} \bar{x}(t)=\left[A \bar{x}(t)+\frac{1}{\lambda} B B^{\tau} \bar{y}(t)+\frac{1}{\lambda} B \bar{z}(t)\right] \mathrm{d} t+\left[C \bar{x}(t)+\frac{1}{\lambda} B^{\tau} \bar{y}(t)+\frac{1}{\lambda} \bar{z}(t)\right] \mathrm{d} W(t), \\
-\mathrm{d} \bar{y}(t)=\left[R \bar{x}(t)+A^{\tau} \bar{y}(t)+C^{\tau} \bar{z}(t)\right] \mathrm{d} t-\bar{z}(t) \mathrm{d} W(t), \\
\bar{x}(0)=x, \quad \bar{y}(T)=Q \bar{x}(T) .
\end{array}\right.
$$

Although the usual monotonicity conditions (see (H3) for example) doesn't hold for the coefficients of the above FBSDE, but the equivalent cost functional method provides an existence and uniqueness result by Theorem 4.4. (ii). In the case that $\lambda=0$, the Hamiltonian system can be represented by the following FBSDE (where $\bar{x}(\cdot)$, $\bar{y}(\cdot), \bar{u}(\cdot)$ are the three unknown processes):

$$
\left\{\begin{array}{l}
\mathrm{d} \bar{x}(t)=[A \bar{x}(t)+B \bar{u}(t)] \mathrm{d} t+[C \bar{x}(t)+\bar{u}(t)] \mathrm{d} W(t), \\
-\mathrm{d} \bar{y}(t)=\left[R \bar{x}(t)+\left(A^{\tau}-C^{\tau} B^{\tau}\right) \bar{y}(t)\right] \mathrm{d} t+B^{\tau} \bar{y}(t) \mathrm{d} W(t), \\
\bar{x}(0)=x, \quad \bar{y}(T)=Q \bar{x}(T) .
\end{array}\right.
$$

The above FBSDE doesn't have a classical form like (3.2), because the diffusion coefficient of backward equation is not $\bar{z}(\cdot)$. To our best knowledge, there are little literature studying on this new type of FBSDE. The equivalent cost functional method gives also an existence and uniqueness result for the new type of FBSDE. 


\subsection{The relation between equivalent cost functional and Riccati equation}

In this subsection, we will give an extension of the family of equivalent cost functionals introduced in the previous subsection, and then we link the extended family of equivalent cost functionals to the classical stochastic Riccati equation.

Let us denote by

$$
\begin{aligned}
\Theta:=\left\{P(\cdot) \in L_{\mathbb{F}}^{\infty}\left(0, T ; \mathcal{S}^{n}\right) \mid\right. & P(t)=P(0)+\int_{0}^{t} \Gamma(s) \mathrm{d} s+\int_{0}^{t} \Lambda(s) \mathrm{d} W(s) \\
& \text { for all } \left.t \in[0, T], \text { where } \Gamma(\cdot), \Lambda(\cdot) \in L_{\mathbb{F}}^{\infty}\left(0, T ; \mathcal{S}^{n}\right)\right\} .
\end{aligned}
$$

Similar to the previous subsection, for any $P(\cdot) \in \Theta$, we apply Itô's formula to $\langle P(\cdot) x(\cdot), x(\cdot)\rangle$ on the interval $[0, T]$ :

$$
\begin{aligned}
-\langle P(0) x, x\rangle= & \mathbb{E} \int_{0}^{T}\left[\left\langle\left(\Gamma+P A+A^{\tau} P+\Lambda C+C^{\tau} \Lambda+C^{\tau} P C\right) x(t), x(t)\right\rangle\right. \\
& \left.+2\left\langle\left(P B+\Lambda D+C^{\tau} P D\right) u(t), x(t)\right\rangle+\left\langle D^{\tau} P D u(t), u(t)\right\rangle\right] \mathrm{d} t \\
& -\mathbb{E}[\langle P(T) x(T), x(T)\rangle] .
\end{aligned}
$$

We introduce another family of equivalent functionals $J^{P}(\cdot), P(\cdot) \in \Theta$ defined as

$$
\begin{aligned}
J^{P}(u(\cdot)):= & J(u(\cdot))-\frac{1}{2}\langle P(0) x, x\rangle \\
= & \frac{1}{2} \mathbb{E} \int_{0}^{T}\left[\left\langle R^{P}(t) x(t), x(t)\right\rangle+2\left\langle S^{P}(t) u(t), x(t)\right\rangle+\left\langle N^{P}(t) u(t), u(t)\right\rangle\right] \mathrm{d} t \\
& +\frac{1}{2} \mathbb{E}\left[\left\langle Q^{P} x(T), x(T)\right\rangle\right],
\end{aligned}
$$

where

$$
\begin{aligned}
R^{P} & =R+\Gamma+P A+A^{\tau} P+\Lambda C+C^{\tau} \Lambda+C^{\tau} P C, \\
S^{P} & =S+P B+\Lambda D+C^{\tau} P D, \\
N^{P} & =N+D^{\tau} P D, \\
Q^{P} & =Q-P(T) .
\end{aligned}
$$

For any $P(\cdot) \in \Theta$, the Hamiltonian system associated with LQ Problem $\left(J^{P}\right)$ is

$$
\left\{\begin{array}{l}
0=\left(S^{P}\right)^{\tau} \bar{x}^{P}(t)+B^{\tau} \bar{y}^{P}(t)+D^{\tau} \bar{z}^{P}(t)+N^{P} \bar{u}^{P}(t) \\
\mathrm{d} \bar{x}^{P}(t)=\left[A \bar{x}^{P}(t)+B \bar{u}^{P}(t)\right] \mathrm{d} t+\left[C \bar{x}^{P}(t)+D \bar{u}^{P}(t)\right] \mathrm{d} W(t) \\
-\mathrm{d} \bar{y}^{P}(t)=\left[R^{P} \bar{x}^{P}(t)+A^{\tau} \bar{y}^{P}(t)+C^{\tau} \bar{z}^{P}(t)+S^{P} \bar{u}^{P}(t)\right] \mathrm{d} t-\bar{z}^{P}(t) \mathrm{d} W(t) \\
\bar{x}^{P}(0)=x, \quad \bar{y}^{P}(T)=Q^{P} \bar{x}^{P}(T)
\end{array}\right.
$$

Lemma 4.7. For all $P(\cdot) \in \Theta$, the existence and uniqueness of Hamiltonian systems (4.7) are equivalent. Moreover, when the Hamiltonian systems (4.7) admit unique solutions $\left(\bar{x}^{P}(\cdot), \bar{u}^{P}(\cdot), \bar{y}^{P}(\cdot), \bar{z}^{P}(\cdot)\right)$, then $\left(\bar{x}^{P}(\cdot), \bar{u}^{P}(\cdot)\right)=(\bar{x}(\cdot), \bar{u}(\cdot))$.

Proof. The proof is similar with that of Lemma 4.3, and we only need replace the invertible transformation (4.4) with the following one

$$
\begin{aligned}
& \bar{x}^{P}(\cdot)=\bar{x}(\cdot), \\
& \bar{u}^{P}(\cdot)=\bar{u}(\cdot) \\
& \bar{y}^{P}(\cdot)=\bar{y}(\cdot)-P \bar{x}(\cdot), \\
& \bar{z}^{P}(\cdot)=\bar{z}(\cdot)-(\Lambda+P C) \bar{x}(\cdot)-P D \bar{u}(\cdot),
\end{aligned}
$$

which is also invertible. 
Theorem 4.8. If there exists a $P_{0}(\cdot) \in \Theta$ such that, $\left(R^{P_{0}}, S^{P_{0}}, N^{P_{0}}, Q^{P_{0}}\right)$ satisfies Assumption (STN), then for any $P(\cdot) \in \Theta$, the stochastic Hamiltonian system (4.7) admits a unique solution $\left(\bar{x}^{P}(\cdot), \bar{u}^{P}(\cdot), \bar{y}^{P}(\cdot)\right.$, $\left.\bar{z}^{P}(\cdot)\right) \in L_{\mathbb{F}}^{2}\left(0, T ; \mathbb{R}^{n}\right) \times \mathcal{U}_{a d} \times L_{\mathbb{F}}^{2}\left(0, T ; \mathbb{R}^{n}\right) \times L_{\mathbb{F}}^{2}\left(0, T ; \mathbb{R}^{n}\right)$. Moreover, $\left(\bar{x}^{P}(\cdot), \bar{u}^{P}(\cdot)\right)$ is the unique optimal pair of $L Q$ Problem $\left(J^{P}\right)$.

The proof is the same as that of Theorem 4.4, and we omit it.

Example 4.9. We assume that a pair of processes $(P(\cdot), \Lambda(\cdot)) \in L_{\mathbb{F}}^{\infty}\left(0, T ; \mathcal{S}^{n}\right) \times L_{\mathbb{F}}^{\infty}\left(0, T ; \mathcal{S}^{n}\right)$ satisfies the following stochastic Riccati equation:

$$
\left\{\begin{aligned}
d P(t)= & \left\{-\left(P A+A^{\tau} P+\Lambda C+C^{\tau} \Lambda+C^{\tau} P C+R\right)\right. \\
& +\left(S+P B+\Lambda D+C^{\tau} P D\right)\left(N+D^{\tau} P D\right)^{-1} \\
& \left.\times\left(S^{\tau}+B^{\tau} P+D^{\tau} \Lambda+D^{\tau} P C\right)\right\} \mathrm{d} t+\Lambda \mathrm{d} W(t) \\
P(T)= & Q \\
K(t):= & N+D^{\tau} P D \in L_{\mathbb{F}}^{\infty}\left(0, T ; \hat{\mathcal{S}}_{+}^{n}\right) \text { and } K^{-1} \text { is bounded also. }
\end{aligned}\right.
$$

For more details about the stochastic Riccati equation, we refer to $[4,6,16,18,19]$. For the first process of solution $P(\cdot)$ of $(4.9)$, we consider the equivalent cost functional $J^{P}(\cdot)$. We have $Q^{P}=0, N^{P}=N+D^{\tau} P D$, $S^{P}=S+P B+\Lambda D+C^{\tau} P D$ and $R^{P}=S^{P}\left(N^{P}\right)^{-1}\left(S^{P}\right)^{\tau}$. Assumption (STN) holds true for $\left(Q^{P}, R^{P}, S^{P}, N^{P}\right)$. And then LQ Problem $(J)$ admits a unique optimal pair $(\bar{x}(\cdot), \bar{u}(\cdot))=\left(\bar{x}^{P}(\cdot), \bar{u}^{P}(\cdot)\right) \in L_{\mathbb{F}}^{2}\left(0, T ; \mathbb{R}^{n}\right) \times \mathcal{U}_{a d}$, which is defined by the following Hamiltonian system:

$$
\left\{\begin{array}{l}
0=\left(S^{P}\right)^{\tau} \bar{x}^{P}(t)+B^{\tau} \bar{y}^{P}(t)+D^{\tau} \bar{z}^{P}(t)+N^{P} \bar{u}^{P}(t), \\
\mathrm{d} \bar{x}^{P}(t)=\left[A \bar{x}^{P}(t)+B \bar{u}^{P}(t)\right] \mathrm{d} t+\left[C \bar{x}^{P}(t)+D \bar{u}^{P}(t)\right] \mathrm{d} W(t), \\
-\mathrm{d} \bar{y}^{P}(t)=\left[S^{P}\left(N^{P}\right)^{-1}\left(S^{P}\right)^{\tau} \bar{x}^{P}(t)+A^{\tau} \bar{y}^{P}(t)+C^{\tau} \bar{z}^{P}(t)+S^{P} \bar{u}^{P}(t)\right] \mathrm{d} t-\bar{z}^{P}(t) \mathrm{d} W(t), \\
\bar{x}^{P}(0)=x, \quad \bar{y}^{P}(T)=0 .
\end{array}\right.
$$

Moreover, we can find the unique solution of the above Hamiltonian system is $\bar{y}^{P}(\cdot)=\bar{z}^{P}(\cdot)=0, \bar{u}^{P}(\cdot)=$ $-\left(N^{P}\right)^{-1}\left(S^{P}\right)^{\tau} \bar{x}^{P}(\cdot)$, and $\bar{x}^{P}(\cdot)$ is defined by the following linear SDE:

$$
\left\{\begin{array}{l}
\mathrm{d} \bar{x}^{P}(t)=\left[A-B\left(N^{P}\right)^{-1}\left(S^{P}\right)^{\tau}\right] \bar{x}^{P}(t) \mathrm{d} t+\left[C-D\left(N^{P}\right)^{-1}\left(S^{P}\right)^{\tau}\right] \bar{x}^{P}(t) \mathrm{d} W(t), \\
\bar{x}^{P}(0)=x .
\end{array}\right.
$$

We notice that the unique optimal control $\bar{u}^{P}(\cdot)$ has a linear feedback form.

By the method of completion of squares, a stochastic LQ problem is well-posed if there exist solutions to the stochastic Riccati equation. Therefore, the stochastic LQ problem is reduced to solve the stochastic Riccati equation. The stochastic version of Riccati equation (4.9) is quite different from the conventional one arising in the deterministic LQ problems. However, due to the presence of the quadratic term in the Riccati equation, it is very difficult to solve. When $S \equiv 0$, and the control weight cost $N$ is positive definite, the solvability of stochastic Reccati equation was an open problem proposed by Bismut [3] and Peng [13] respectively. Tang [16] obtained a general existence and uniqueness result, and solved this open problem. When the control weight cost $N$ is indefinite, Chen et al. [6], Theorem 4.6, gave a necessary and sufficient condition for the existence and uniqueness of Riccati equation (4.9) when $C \equiv 0, S \equiv 0$ and all the other matrices $A, B, D, R, N, Q$ are deterministic. In [5], Chen and Zhou continued to keep the deterministic framework, but got rid of the restriction $C \equiv 0$. They proved a sufficient condition for the solvability of Riccati equation where the control weight cost 
is nonnegative. As for the indefinite case, they were still not able to prove the solvability of Riccati equation, but they gave a necessary condition for it.

On the other hand, Example 4.9 shows that the classical Riccati equation can be associated with a special equivalent cost functional. Therefore, in dealing with LQ problems with indefinite control weight costs, the equivalent cost functional method is more extensible than the stochastic Riccati equation method. And the advantage of the new method is that one can avoid to solve the Riccati equation, by looking for other more flexible equivalent cost functionals, such as $J^{P}(\cdot)$ defined by (4.6), $J^{f}(\cdot)$ defined by (4.2), etc.

Remark 4.10. In this subsection, we show that when the Riccati equation is solvable, then by an equivalent cost functional with Assumption (STN), the corresponding Hamiltonian system is solvable also. However, the excellent work of Tang [16] provided a rigorous derivation of the existence of Riccati equation from that of Hamiltonian system for LQ problems with positive control weight costs and $S \equiv 0$. In the more general case where the control weight costs may be indefinite and $S$ may not be zero, the similar result can also be obtained under some extra assumptions in the author's viewpoint. However, the detailed derivation and the specific assumptions remain open to discussion at this stage of our investigation. Since the equivalent cost functional method can bring some new existence results of Hamiltonian system, so it seems to provide a promising new approach to the study of the existence of stochastic Riccati equation.

\section{Conclusion}

In this paper, we proposed a new method named the equivalent cost functional method, and we apply it to deal with the LQ problems with indefinite control weight costs. Compared to the classical methods, the new method is simple, flexible, and non-abstract. As a by-product, the new method can be used to study the solvability of the stochastic Hamiltonian systems or FBSDEs.

Although we focus on the stochastic LQ optimal control problems, the new method can be used to develop some results for nonlinear stochastic optimal control and nonlinear stochastic differential game problems. In Jeanblanc and $\mathrm{Yu}[9]$, the equivalent cost functional method is applied to deal with a utility optimization problem arising from mathematics finance.

Acknowledgements. The author would like to thank Associate Editor and two anonymous referees for their constructive and insightful comments for improving the quality of this work, and Prof. Zhen Wu for many helpful discussions and suggestions.

\section{REFERENCES}

[1] B.D.O. Anderson and J.B. Moore, Optimal control-Linear quadratic methods. Prentice-Hall, New York (1989).

[2] A. Bensoussan, Lecture on stochastic cntrol, Part I, in Nonlinear Filtering and Stochastic Control, Lecture Notes in Math. 972. Springer-Verlag, Berlin (1983) 1-39.

[3] J.M. Bismut, Controle des systems linears quadratiques: applications de l'integrale stochastique, in Séminaire de Probabilités XII, Lecture Notes in Math. 649, edited by C. Dellacherie, P.A. Meyer and M. Weil. Springer-Verlag, Berlin (1978) $180-264$.

[4] S. Chen and J. Yong, Stochastic linear quadratic optimal control problems. Appl. Math. Optim. 43 (2001) $21-45$.

[5] S. Chen and Z. Zhou, Stochastic linaer quadratic regulators with indefinite control weight costs. II. SIAM J. Control Optim. 39 (2000) 1065-1081.

[6] S. Chen, X. Li and X. Zhou, Stochastic linear quadratic regulators with indefinite control weight costs. SIAM J. Control Optim. 36 (1998) 1685-1702.

[7] M.H.A. Davis, Linear estimation and stochastic control. Chapman and Hall, London (1977).

[8] Y. Hu and S. Peng, Solution of forward-backward stochastic differential equations. Prob. Theory Relat. Fields 103 (1995) $273-283$.

[9] M. Jeanblanc and Z. Yu , Optimal investment problems with uncertain time horizon. Working paper.

[10] R.E. Kalman, Contributions to the theory of optimal control. Bol. Soc. Math. Mexicana 5 (1960) 102-119. 
[11] J. Ma and J. Yong, Forward-backward stochastic differential equations and their applications, Lecture Notes in Math. 1702. Springer-Verlag, New York (1999).

[12] S. Peng, New development in stochastic maximum principle and related backward stochastic differential equations, in proceedings of 31st CDC Conference. Tucson (1992).

[13] S. Peng, Open problems on backward stochastic differential equations, in Control of Distributed Parameter and Stochastic Systems (Hangzhou, 1998). edited by S. Chen et al., Kluwer Academic Publishers, Boston (1999) 966-979.

[14] S. Peng and Z. Wu, Fully coupled forward-backward stochastic differential equation and applications to optimal control. SIAM J. Control Optim. 37 (1999) 825-843.

[15] R.T. Rockafellar, Convex Analysis. Princeton University Press, Princeton, New Jersey (1970).

[16] S. Tang, General linear quadratic optimal stochastic control problems with random coefficients: linear stochastic Hamilton systems and backward stochastic Riccati equations. SIAM J. Control Optim. 42 (2003) 53-75.

[17] W.M. Wonham, On a matrix Riccati equation of stochastic control. SIAM J. Control Optim. 6 (1968) 312-326 .

[18] Z. Wu, Forward-backward stochastic differential equations, linear quadratic stochastic optimal control and nonzero sum differential games. Journal of Systems Science and Complexity 18 (2005) 179-192.

[19] J. Yong and X. Zhou, Stochastic controls: Hamiltonian systems and HJB equations. Springer-Verlag, New York (1999). 